\title{
Laryngo-Tracheal Displacement and Respiratory Distress in an Infant with Congenital Thymic Hyperplasia
}

\author{
Michael Wolff, ${ }^{1}$ Süha Demirakca, ${ }^{1}$ Anna Kristina Kilian, ${ }^{2}$ \\ Horst Schroten, ${ }^{1}$ and Tobias Tenenbaum ${ }^{1}$ \\ ${ }^{1}$ University Children's Hospital, Medical Faculty Mannheim, Heidelberg University, Theodor-Kutzer-Ufer 1-3, \\ 68167 Mannheim, Germany \\ ${ }^{2}$ Department of Radiology, Medical Faculty Mannheim, Heidelberg University, Theodor-Kutzer-Ufer 1-3, 68167 Mannheim, Germany
}

Correspondence should be addressed to Tobias Tenenbaum, tobias.tenenbaum@umm.de

Received 14 February 2011; Accepted 3 April 2011

Academic Editors: N. P. Juffermans and B. A. Rybicki

Copyright () 2011 Michael Wolff et al. This is an open access article distributed under the Creative Commons Attribution License, which permits unrestricted use, distribution, and reproduction in any medium, provided the original work is properly cited.

Thymic hyperplasia is usually an asymptomatic condition observed in infancy. A five-week-old boy presented with respiratory distress and feeding disorder, in which chest X-ray and bronchoscopy revealed a strong laryngotracheal displacement. Sonography and MRI confirmed the diagnosis of a thymic hyperplasia. Corticosteroid therapy led to improvement of clinical symptoms. Thymic hyperplasia may lead to laryngo-tracheal displacement and respiratory distress in neonates and young children.

\section{Introduction}

Although thymic enlargement is a common and physiological finding on X-rays of children and neonates, symptomatic hyperplasia is a very rare condition. In addition to X-ray, ultrasound, CT, and MRI have been used to confirm the diagnosis in difficult cases $[1,2]$. Treatment modalities of symptomatic patients are still controversial and corticosteroid therapy for symptomatic thymic hyperplasia has not been proven to be successful so far [3]. This report represents a unique case with a symptomatic thymic hyperplasia and a strong laryngo-tracheal deviation, demonstrated by chest X-ray and bronchoscopy, that could successfully be treated with corticosteroids.

\section{Case Report}

We report of a five-weeks-old infant presenting at our hospital with a history of inspiratory stridor and feeding disorder since birth. Pregnancy and birth were uneventful. Clinical examination has shown besides the inspiratory stridor, dyspnea, and tachypnea no other pathologies. Blood results revealed a normal blood count, electrolytes, liver and kidney function values and blood coagulation. Blood gases and oxygen saturation were normal, too. The initial X-ray shown in Figure 1(a) showed a significant displacement of the trachea to the left by a big mass in the upper thoracic aperture. By ultrasound we made the presumptive diagnosis of a thymic hyperplasia. During inspiration the thymus moved cranial, which was accompanied by an inspiratory stridor. No thyroid or lymph node enlargement could be detected. The additional MRI scan confirmed a spaceoccupying lesion in the upper thoracic aperture with a density similar to thymic tissue and a maximum width of $3.2 \mathrm{~cm}$ (Figure 1(b)). The trachea was not narrowed (Figure 1(c)). The bronchoscopy showed strong a bend of the larynx and the trachea to the left, but no other abnormalities.

Since the child remained in respiratory distress and was difficult to feed we decided to perform a corticosteroid therapy. We started a therapy with $4 \mathrm{mg} / \mathrm{kg}$ methylprednisolone for one week (the child weighed $4,5 \mathrm{~kg}$ ), reduced stepwise and stopped after four weeks, the last dose being $1 \mathrm{mg}$ once daily. No corticosteroid side effects as hypertension, glucosuria, and electrolyte disturbances have been observed. Clinically the inspiratory stridor, the tachydyspnoe, and feeding disorder disappeared after four days of treatment. Two months after presentation we performed a second chest X-ray (Figure $1(\mathrm{~d})$ ) showing an anatomic normal trachea 


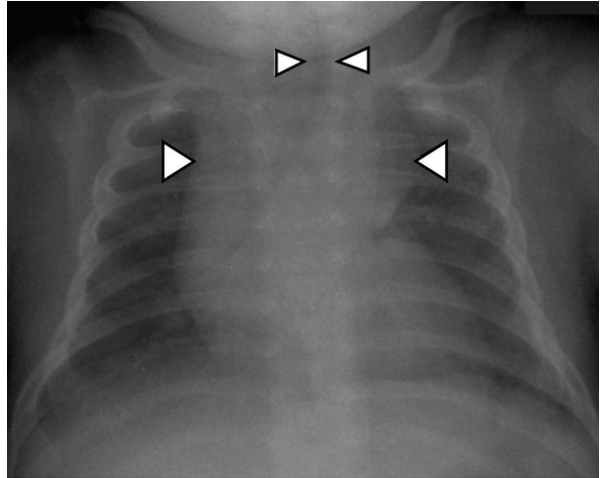

(a)

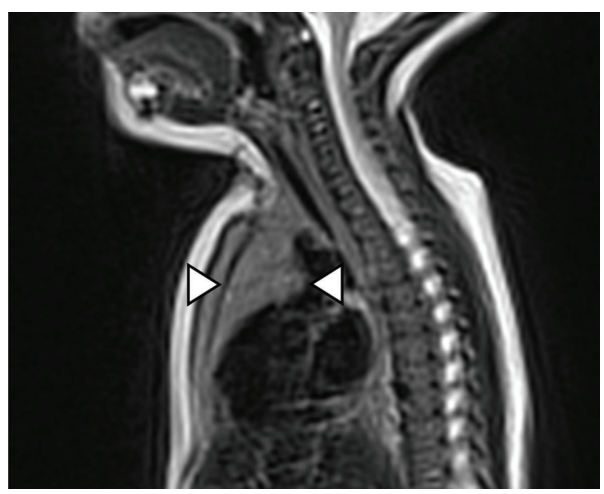

(c)

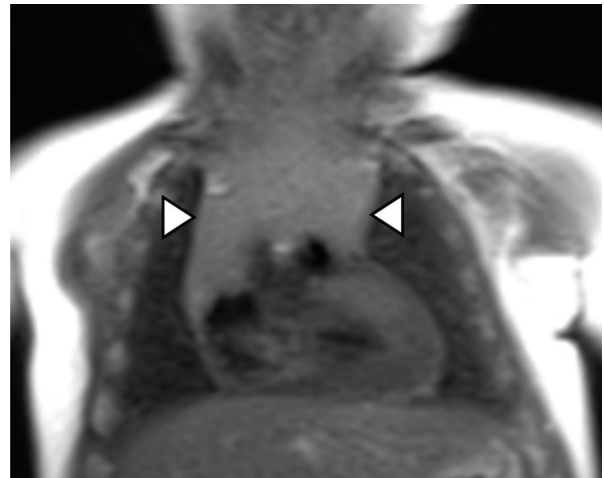

(b)

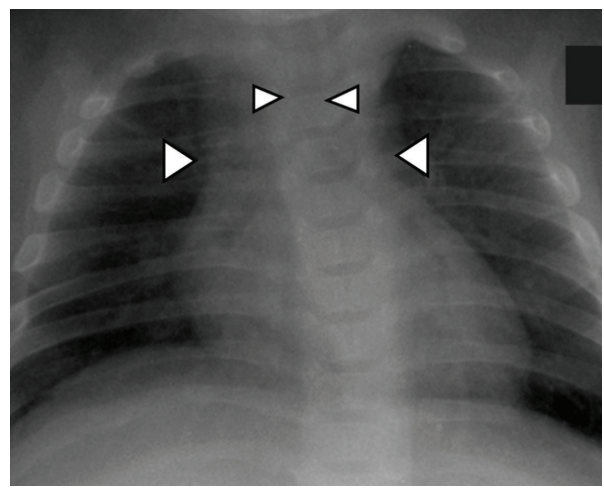

(d)

Figure 1: (a) Chest X-ray of the infant at presentation. Note the large, well-defined radiopaque mass at the upper mediastinum (big white arrows) dislocates the trachea (small white arrows) to the left. (b) Coronal T2-weighted HASTE (half fourier-acquired single shot turbo spin echo) image showing thymic enlargement (white arrows). (c) Sagittal T2-weighted BLADE/PROPELLER (periodically rotated overlapping parallel lines with enhanced reconstruction) image with a respiratory compensation technique for motion correction showing retrosternal distension (white arrows). (d) Chest X-ray of the infant 2 months after therapy. No dislocation of the trachea (white arrows) could be observed.

without any tracheal dislocation or obstruction. Sonographically there was also a clear reduction of thymic tissue. In the clinical followup the child showed no recurrence of his symptoms 1, 2, and 7 months after therapy.

\section{Discussion}

Although thymic enlargement is a common and physiological finding on X-rays of children and neonates, symptomatic hyperplasia is a very rare condition. The condition of true thymic hyperplasia is defined as an increase of both size and weight of the gland while it maintains normal microscopic architecture. There exist three different forms. First the true thymic hyperplasia without any underlying disease, second as a form of rebound phenomenon caused from steroid therapy, chemotherapy, growth hormone treatment or severe stress and third associated with endocrine abnormalities, sarcoidosis, myasthenia gravis (MG) and Beckwith-Wiedeman syndrome (BWS) [4].

Most mediastinal tumors in the pediatric population are either neurogenic in origin (33\%) or lymphomas (41\%). However, primary thymic lesions (such as thymic cysts, thymolipomas, and thymic hyperplasia) represent only $2.5 \%$ of mediastinal tumors, while thymomas comprise about $1 \%$ and typically have an aggressive course and poor survival [5]. In our case no biopsy has been taken, because age and blood results as well as the MRI morphology made a lymphoma rather unlikely.

The thymus can present as a confusing mass-like density as its size, shape, position, and growth pattern are variable. Performance of a chest X-ray and an ultrasound of the thymus and in some case also MRI or CT scan may be necessary to secure the diagnosis of thymic hyperplasia and to exclude malignancy $[1,2]$. Especially the ultrasound is an easily accessible diagnostic tool, that shows a typical morphology and is also able to demonstrate correlation with inspiratory stridor with cranial movement of the hypertrophic thymus $[1,2]$.

If the morphological diagnostic imaging is in favor of this diagnosis of thymic hyperplasia especially in symptomatic infants a trial with corticosteroids, and a close followup as first-line therapy may be considered [1]. Corticosteroids are potent to reduce the volume of the thymus and achieve its partial involution and thereby may prevent invasive surgery in symptomatic patients [3]. Short- and long-term application of corticosteroids has been demonstrated to cause 
thymic involution [6]. However, the current literature about a possible beneficial of corticosteroids in thymic hyperplasia is still controversial [3]. Some recommend a stress test to aid in making the correct diagnosis, as oral administration of steroids will usually result in involution of a thymic mass. Unfortunately, the test is not specific and lymphatic tumours will also involute. Corticosteroid therapy requires a long time followup because a relapse of thymus hyperplasia after natural or iatrogenic steroid excess may occur. Seven month after therapy our patient remained asymptomatic.

Still surgery may be necessary when the mass enlarges and may affect pulmonary development or the symptoms cannot be controlled otherwise. Patients have been reported who required surgical thymectomy or even additional pulmonary lobectomy or emergency surgery in conditions like a bleeding intrathoracical thymic cysts [7]. However, others have suggested that all unknown mediastinal masses must be removed by surgery $[8]$.

Our case of a child with thymic hyperplasia demonstrates that a strong laryngo-tracheal displacement can lead to respiratory distress and feeding disorder in children.

\section{References}

[1] L. A. Parker, G. Gaisie, and J. H. Scatliff, "Computerized tomography and ultrasonographic findings in massive thymic hyperplasia. Case discussion and review of current concepts," Clinical Pediatrics, vol. 24, no. 2, pp. 90-94, 1985.

[2] L. M. Linde, "Normal thymus simulating pericardial disease: diagnostic value of magnetic resonance imaging," Pediatrics, vol. 88 , no. 2, p. 328, 1991.

[3] E. R. Sauter, R. M. Arensman, and K. W. Falterman, "Thymic enlargement in children," American Surgeon, vol. 57, no. 1, pp. 21-23, 1991.

[4] W. J. Hofmann, P. Möller, and H. F. Otto, "Thymic Hyperplasia. I. True thymic hyperplasia. Review of the literature," Klinische Wochenschrift, vol. 65, no. 2, pp. 49-52, 1987.

[5] J. L. Grosfeld, M. A. Skinner, F. J. Rescorla, K. W. West, and L. R. Scherer, "Mediastinal tumors in children: experience with 196 cases," Annals of Surgical Oncology, vol. 1, no. 2, pp. 121-127, 1994.

[6] P. M. Lundin and U. Schelin, "The effect of steroids on the histology and ultrastructure of lymphoid tissue. 3. Thymus in prolonged steroid induced involution," Pathologia Europaea, vol. 4, no. 1, pp. 58-68, 1969.

[7] F. Eifinger, K. Ernestus, G. Benz-Bohm et al., "True thymic hyperplasia associated with severe thymic cyst bleeding in a newborn: case report and review of the literature," Annals of Diagnostic Pathology, vol. 11, no. 5, pp. 358-362, 2007.

[8] A. G. Linegar, J. A. Odell, W. M. Pitt Fennell et al., "Massive thymic hyperplasia," Annals of Thoracic Surgery, vol. 55, no. 5, pp. 1197-1201, 1993. 


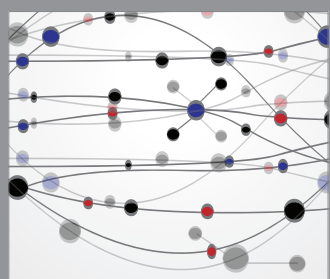

The Scientific World Journal
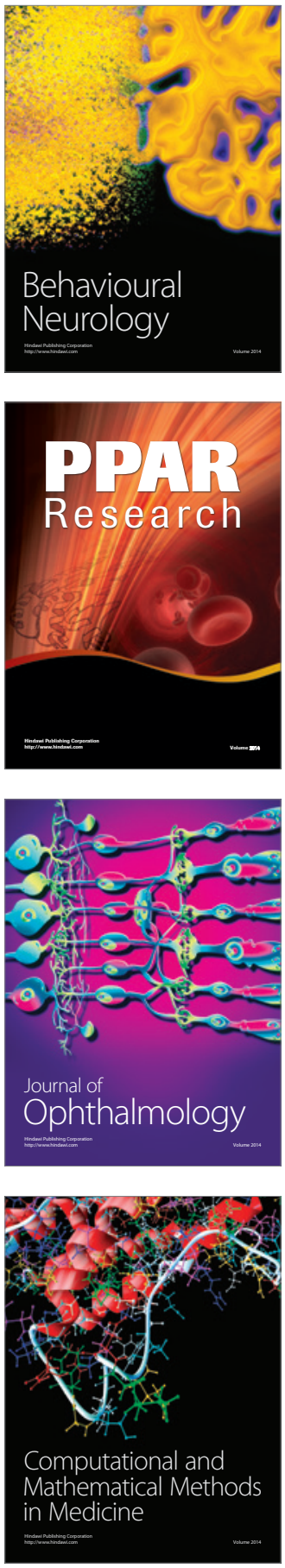

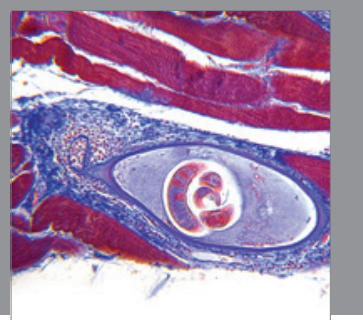

Gastroenterology

Research and Practice
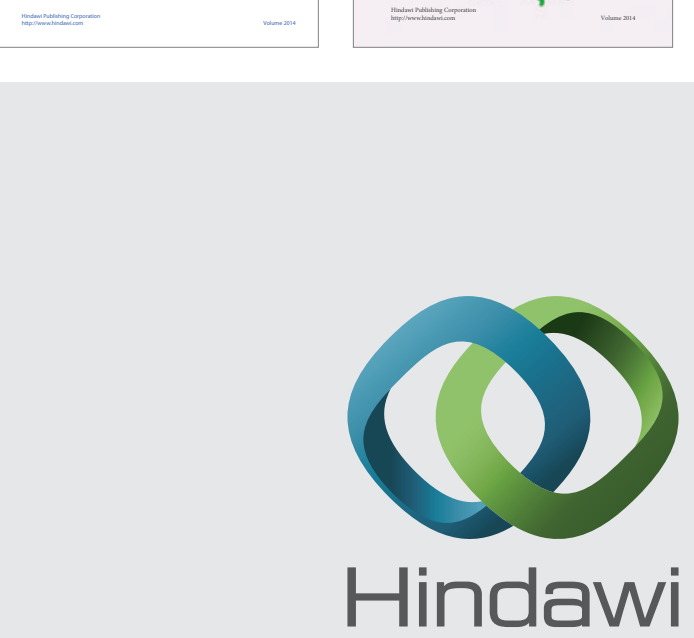

Submit your manuscripts at

http://www.hindawi.com
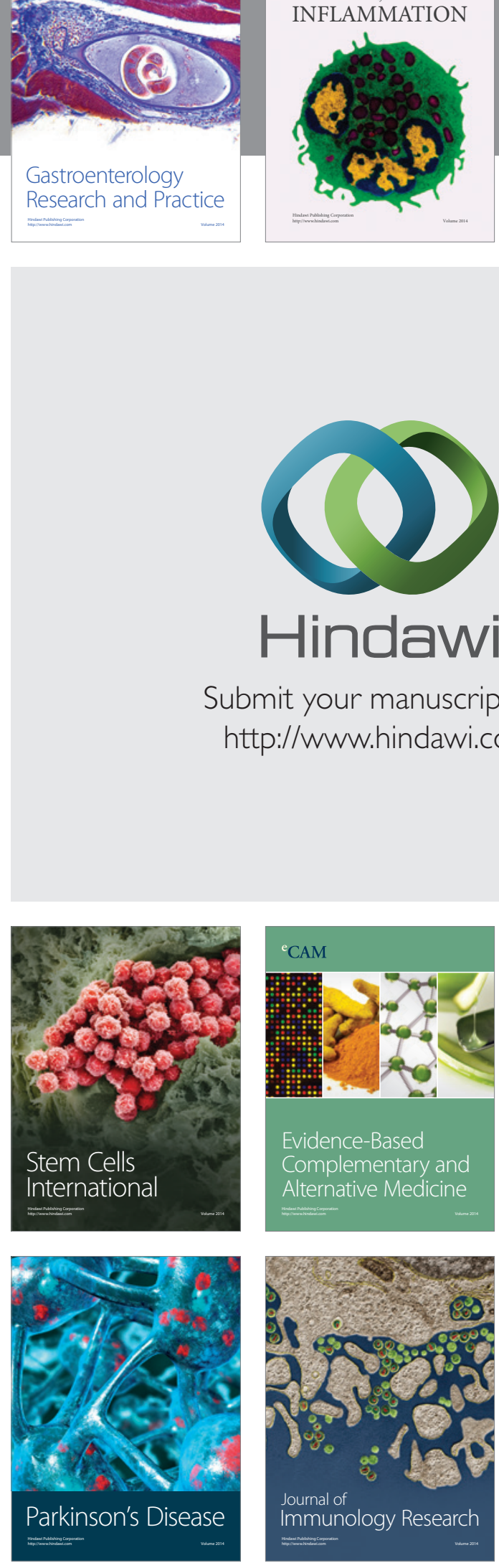

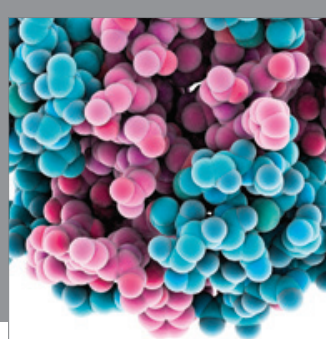

Diabetes Research
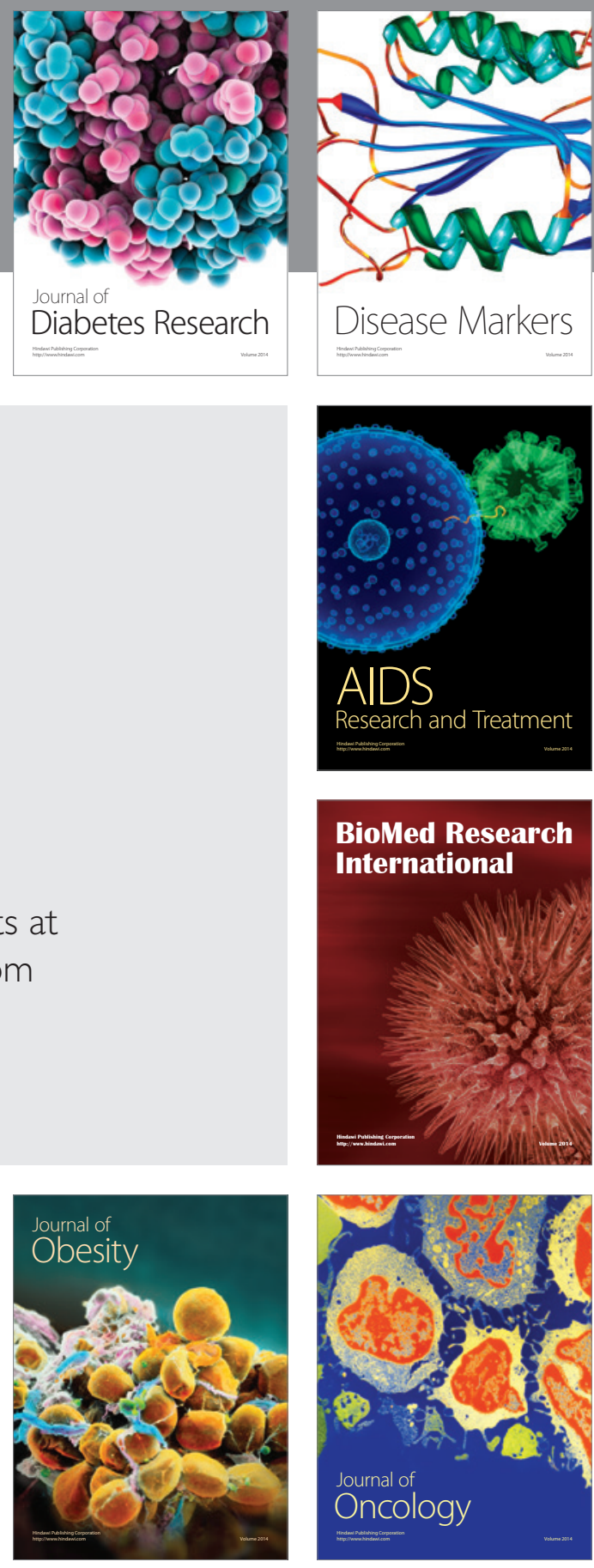

Disease Markers

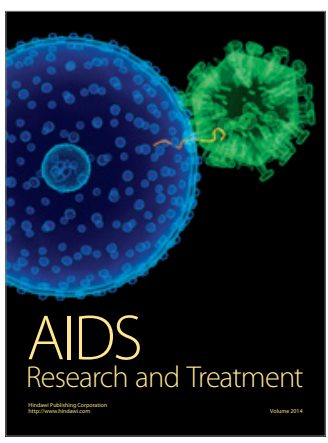

BioMed Research

International
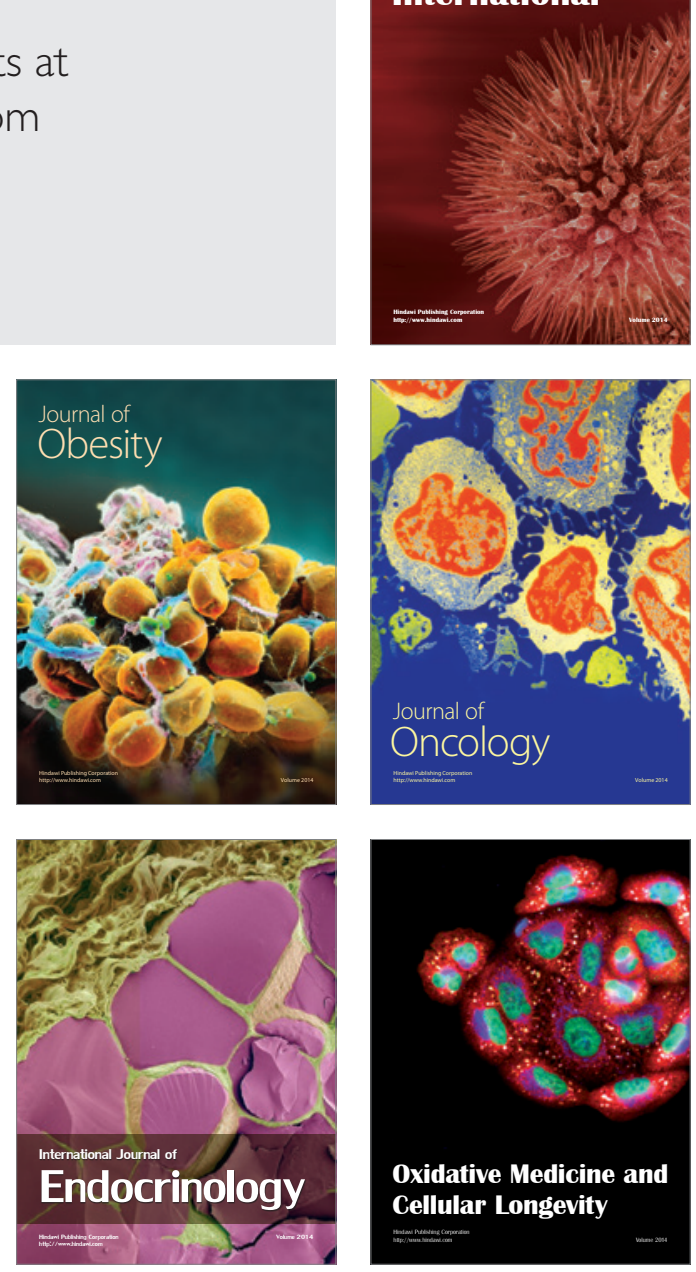